\title{
The effect of tray selection and viscosity of impression materials on the accuracy of dies produced from dual arch impressions - An in-vitro study
}

\author{
Dr. Pramod Sankar.S ${ }^{1}$, Dr. Menon Prasad Rajagopal ${ }^{2}$, Dr. Rajitha A.V ${ }^{3}$ \\ $\left({ }^{1}\right.$ Assistant Professor, Department of Prosthodontics Educare institute of Dental Sciences/ KUHS, India ) \\ ( ${ }^{2}$ Professor and HOD, Department of Prosthodontics Educare institute of Dental Sciences/KUHS, India ) \\ $\left({ }^{3}\right.$ Senior Resident, Department of OMR, Govt. Dental College, Kozhikode/KUHS, India)
}

\begin{abstract}
Objectives :The aim of this in-vitro study was to compare the accuracy of dies generated from metal and plastic dual arch trays impressions using two different viscosities of polyvinyl siloxane impression material and comparing the results to that obtained using a conventional full arch custom impression technique.

Materials and method: Seven set of impressions were made of an experiment model using different combinations of, dual arch trays and viscosities of impression materials and the dimensions of the dies thus obtained were compared with those obtained from conventional full arch custom arch tray and medium viscosity impression material.

Results: The gypsum dies prepared, from the complete arch custom tray technique and the dual arch impression technique using plastic and metal dual arch trays were generally larger in bucco-lingual dimension and shorter in mesio-distal dimension.

Conclusions: All the seven types of impression techniques produced clinically successful impressions of standard brass die and dies so developed were within clinical standards to produced clinically successful restorations.
\end{abstract}

Key Words: Closed mouth impression Dual arch tray, Impressions, Triple tray.

\section{Introduction}

Dual arch impression technique is a closed mouth technique, where a single, double sided impression is made, to simultaneously imprint the prepared tooth, its adjacent teeth and the opposing tooth segment, in their normal physiological position of maximum intercuspation. The trays used are specially designed with metal or plastic flanges, spanned by a mesh fabric or paper inserts dividing the trays into upper and lower compartments. The dual arch impression technique is indicated for the single posterior indirect restorations and short span fixed partial dentures where a stable maximal intercuspal position and mutually protected occlusion exists. The major advantage of this technique is reduction in error and need for occlusal adjustment. Other proposed advantages are saving time and material, patient comfort, ease of use and simultaneous record of the prepared tooth, opposing teeth, interocclusal relation that needs three different steps in a conventional impression technique.

However, the lack of rigidity[3,5-6] of a dual arch tray is considered to be a drawback which may lead to flexure of the tray impression complex, and the resulting rebound could give inaccurate die dimension and ill fitting restorations. The purpose of this in-vitro study is to compare the accuracy of dies generated from metal and plastic dual arch trays impressions using two different viscosities of polyvinyl siloxane impression material and comparing the results to that obtained using a conventional full arch custom impression technique.

\section{Aims and Objectives}

The objectives of the study were to compare the accuracy of gypsum dies, in three dimensions, produced from

1. Dual arch plastic tray and putty / wash elastomeric impression material - single mix technique.(DPPS)

2. Dual arch plastic tray and putty / wash elastomeric impression material - double mix technique.(DPPD)

3. Dual arch plastic tray and Monophase impression material - single mix technique (DPMS)

4. Dual arch metal tray and putty / wash elastomeric impression material -single mix technique (DMPS)

5. Dual arch metal tray and putty / wash elastomeric impression material - double mix technique. (DMPD)

6. Dual arch metal tray and Monophase impression material -single mix technique. (DMMS)

7. Complete arch custom tray and Monophase impression material. (FCMS). 


\subsection{The experimental model - Fig I}

\section{Materials and method}

The experimental model used was a machined brass die of dimension $(\mathrm{OG}=4.9326 \mathrm{~mm}, \mathrm{MD}=$ $8.1375 \mathrm{~mm}, \mathrm{BL}=8.1005 \mathrm{~mm}$ ). The die was screwed onto the mandibular typhodont (Nissin, Tokyo, Japan) in thEeleft first molar position. The mandibular and maxillary typhodont were articulated in maximal intercuspation on a mean -value articulator using auto-polymerizing acrylic resin as in figure.

\subsection{MaterialsN- Fig II \& III}

a) Dual arch impression trays - Plastic (Caprisons, Mumbai)

b) Dual arch impression trays - Metal (Caprisons, Mumbai)

c) Full arch acrylic custom tray

d) Tray adhesive- universal

e) Addition silicone impression material ( Aquasil, Dentsply)

a. Putty regular set

b. Low viscosity - cartridge

c. Monophase - cartridge

f) Mixing tips (large and small)

g) Auto-mixing gun

\subsection{Impression making- Fig IV}

The trays were evaluated over the typhodont for proper seating, before definitive impressions were made. The dual arch trays should fit passively without impinging on typhodont so that articulator could be closed to the maximum intercuspal position.

One coat of adhesive was applied to the internal surface and borders of the complete arch custom tray, and the walls of dual arch trays 10 minutes before impression making, conforming to the manufacturer's recommendations.

Using the disposable syringe tip attached to the mixing tip of auto mixing cartridges and auto mix gun, around $1.2 \mathrm{ml}$ of low viscosity material was injected around and over the die, and adjacent teeth. Five complete activations of auto mix cartridge were used to deliver $11 \mathrm{ml}$ of monophase into each half of custom arch tray and both sides of dual arch trays. The complete arch custom tray was then seated, until the vertical stops were engaged, and held in place for 5 min according to manufacturers' instruction.

Loaded dual arch tray was placed onto the machined die and articulator closed into maximal intercuspation. A weight of $1.5 \mathrm{~kg}$ is placed over the articulator to simulate the force during maximum intercuspation. Impression was allowed to set for $5 \mathrm{~min}$ according to manufacturer's instruction. The tray was then removed in a single movement, holding both the lingual and buccal flanges, to minimize distortion. The sequence of seven impressions were randomized. A total of seventy impressions, 10 for each group were made.

\subsection{Evaluation of impression}

The impression so obtained was examined for voids, "show through", and other artifacts. "Show through" at a particular point indicates impingement of the tray onto the typhodont, to the extent that the impression material was completely displaced from that area, and possible distortion of the tray during impression procedure. Such impressions were discarded and remade after corrective measures were taken to prevent a recurrence of the problem. To ensure that the articulator was closed to maximum intercuspation, the impressions were held up to a light source to see if occlusal surfaces of all the teeth were covered with a thin layer of impression material and are translucent. Opaque occlusal surface indicates an improper closure and such impressions were remade. The impressions were poured in gypsum 60 min later.

\subsection{Pouring the cast and die fabrication - Fig V}

Die Stone was hand mixed for $5 \mathrm{sec}$ in the ratio of $20 \mathrm{ml}$ distilled water: $100 \mathrm{gm}$ powder, then mixed under vacuum for $30 \mathrm{sec}$, and vibrated into the impressions complying with the manufacturer's instruction.

For dual arch trays, the opposing sides were poured first, followed by the impression side one hour later. The casts were allowed to set for $24 \mathrm{hrs}$ before being removed from the trays. The casts were then sectioned with a diamond disc to fabricate gypsum dies.

\subsection{Measurement of the Standard (Model) and Gypsum dies. - Fig VI}

The brass die and the gypsum dies were measured using Universal Measuring Microscope (Carl Zeiss, Germany). The axial section method was used in this study. This Optico-mechanical method makes use of measuring knife edge moved to the thread so that it can be measured in the axial section directly. This is otherwise possible neither by purely mechanical nor purely optical methods. 
The bases of the working dies were made parallel with the superior surface, so that the positioning of the dies in the microscope could be reproduced. This enabled standardization of the measuring technique. Each die was measured three times for the same dimension and the mean value was taken.

The data thus obtained was tabulated and statistically analyzed.

\section{Results}

Table I, II and III gives the raw data for the occluso-gingival (OG), mesio-distal (MD) and buccolingual (BL) measurements for all the working dies respectively. Table IV gives the standard deviation of the $\mathrm{OG}, \mathrm{MD}$ and $\mathrm{BL}$ values for the different impression groups. Table $\mathrm{V}$ gives the results one way ANOVA of the sample values. p- Values were found to be significant. Table VI, VII and VIII gives the result of Scheffe test done to compare different impression groups with each other in OG, MD, BL values respectively. Graph I, II and III represent categorized plot for OG, MD and BL values respectively.

\section{DPPS and DPPD}

The results can be summarized as follows:

These impression techniques produced dies that were significantly larger in bucco-lingual dimension than the standard, and the dies generated from DMMS and FCMS technique. No significant difference in mesio-distal and occluso-gingival dimension was noted.

\section{DMPS and DMPD}

These impression techniques produced dies that were significantly larger in bucco-lingual dimension than the standard. No significant differences were noted in mesio-distal and occluso-gingival dimensions.

\section{DPMS, DMMS and FCMS}

In mesio-distal dimension, the dies produced from these impression techniques were significantly smaller than the standard brass die. Only the DPMS dies were significantly larger in bucco-lingual dimension than the standard.

\section{Discussion}

In our study, the conventional complete arch custom tray monophase impression technique (FCMS) and metal dual arch with monophase impression material (DMMS) produced dies that were closest to the standard in the bucco-lingual dimension. The dies produced from all other impression techniques were significantly larger from the standard. The dies from dual arch plastic trays with putty as tray material (DPPS and DPPD) were significantly larger than DMMS and FCMS.

The larger bucco-lingual dimension of the working dies could be explained by the tray adhesive factor, used with all the impression trays. During polymerization reaction, the impression material shrinks towards the centre of the mass. The use of the tray adhesive, however, would redirect this shrinkage towards the impression tray walls, resulting in larger dies in the diameter, bucco-lingually explaining the smaller mesio-distal dimension of the working dies.

The reason for dies made from dual arch plastic trays with putty as tray material (DPPS and DPPD ) being significantly larger may be due to the highly rigid set putty material decreasing the rebound of the tray from its flexed state.

In the mesio-distal dimension, statistically significant differences were observed between the three tray types and the standard when monophase impression material (DPMS, DMMS and FCMS) was used. This significantly smaller mesio-distal dimension may be explained by the greater polymerization shrinkage shown by the medium viscosity monophase impression material compared to the lesser shrinkage by the high viscosity Putty impression material.

Even though DPMS and DMMS were significantly lower than the standard (Brass die), there were no statistically significant difference with FCMS, which is considered as the clinical standard in impression making. In the occluso-gingival dimension, the dies from dual arch were generally shorter than the standard. But the difference was not statistically significant. With a dual arch tray, the shrinkage of the impression material towards the tray walls affects only in the bucco-lingual direction as there is only a paper insert separating the tray into upper and lower halves in the occluso-gingival direction. Thus the possible explanation for the shorter occluso-gingival dimension might be pouring the counter impression first so that the weight of the stone causing shorter dies in the occluso-gingival dimension on the working side

The dies from conventional custom arch impression trays were taller than the standard. The tray adhesive factor on the superior surface of the tray may resist polymerization shrinkage towards the centre thus making the die taller. The effect of the weight of the stone by pouring the counter impression first, was also absent here.

The measurements made on the stone casts are potentially affected not only by the impression material and the tray type but also by the expansion of the dental stone used. The International Dental Standards states that $0.15 \%$ is the maximum linear dimensional change of elastomeric impression material. . In this investigation 
the die stone with a setting expansion of $0.08 \%$ (as given by the manufacturer) was used, which may compensate for any dimensional change in the polymerized impression material. The significantly larger dies, in bucco-lingual dimension, obtained from both dual arch trays and custom arch trays were clinically insignificant as it ranged only from $9 \mu \mathrm{m}-26 \mu \mathrm{m}$. The margin is considered to be open only if there is a discrepancy of $50 \mu \mathrm{m}$ or more. $[4,7]$ The openings less than this will not be detected even by a sharp explorer.

The smaller mesio-distal and the occluso-gingival dimension of the working dies obtained would also be insignificant clinically as it can be masked with an extra coat of die spacer on the inter proximal and occlusal

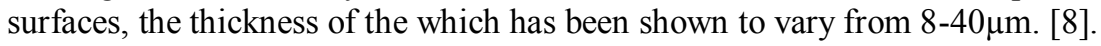

This study did not take into account the effect of dual arch on interocclusal relationships. As seen in study by Parker et al [9], quadrant dual arch impressions technique mounted casts with significantly more accurate maximal intercuspal relationships than casts from full arch impressions. Moreover ,the study focused only on single crown and did not evaluate the accuracy of reproducing short span bridges. But study by Werrin [10] concluded that dual arch impressions produce accurate fitting short span 3 unit FDP's. Yet more prospective trails with larger samples should be carried out to validate such results.

\section{Conclusion}

All the seven types of impression a technique produced clinically successful impressions of standard brass die and the dies so developed were within clinical standards to produce clinically successful restorations. Dual arch impression technique is cost effective and is time and material saving. It can be an accurate method and can be put to use successfully, provided the operator understands the indications and contraindications of the procedure.

\section{References}

[1]. E.G.Wilson, S.R Werrin. Double arch impressions for simplified dentistry. JPD 1983(49)198-202

[2]. Davis R D ,Schwartz R S. Dual- arch and custom -tray impression accuracy.JADA 1991(4)89-92

[3]. Bass E V, Kafalias M C Dual arch impressions Aust Dent J 1992(37)1-5

[4]. Davis R D, Schwartz R S. Marginal adaptation of castings made with dual-arch and custom trays. Am J Dent 1992(5)253-4

[5]. G.J.Kaplowitz. Troubleshooting dual arch impressions. JADA 1996(127):234-40

[6]. Kaplowitz. Trouble shooting dual arch impressions -II. JADA 1997 (128) : 1277- 81.

[7]. COX J R. A clinical study comparing the marginal and occlusal accuracy of crowns fabricated from dual arch and complete arch impressions. Aus Dent.J 2005(50):90-4.

[8]. Jeffrey AC, Glen HJ, Lepe.X. The effect of tray selection, viscosity of impression material, and sequence of pour on the accuracy of dies made from the dual arch impressions. JPD 2003(90): 143-9.

[9]. M.H.Parker, S.M.Cameron, J.C.Hughbanks. comparison of occlusal contacts in maximum intercuspation for two impression techniques. JPD 1997(78):255-9

[10]. S.R.Werrin. The 2- minute impression technique. Quintessence International. 1996(270:179-81.

\section{Figures}

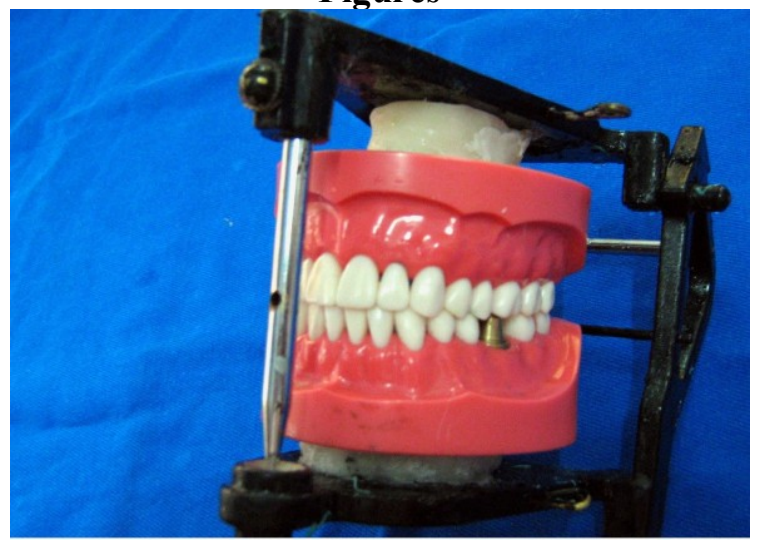

Figure I- Experiment model 


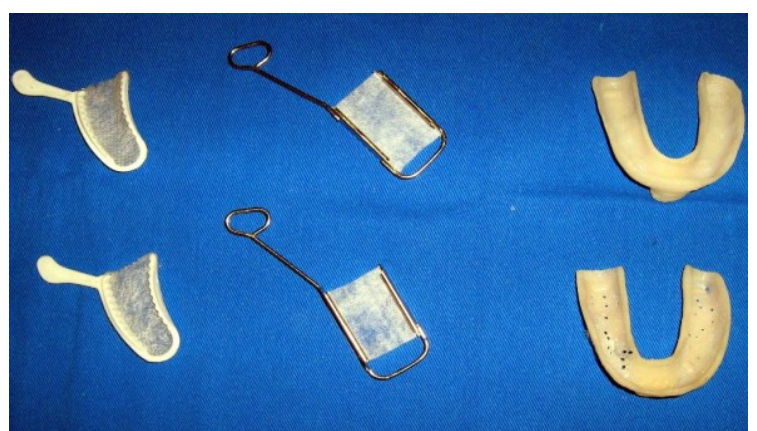

Figure II- Trays used

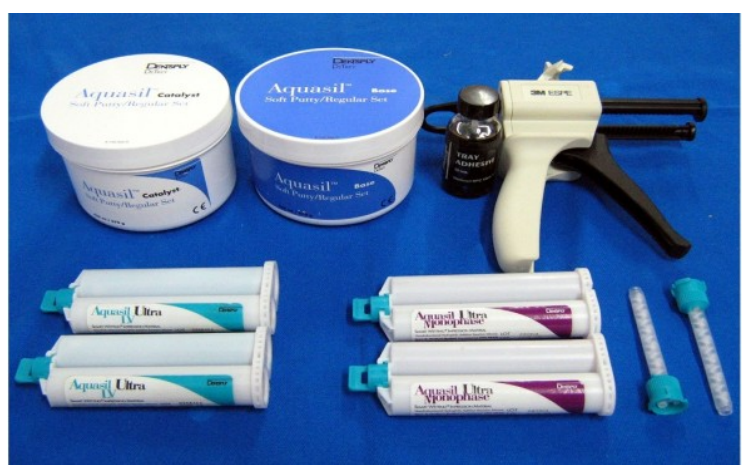

Figure III- Impression material used

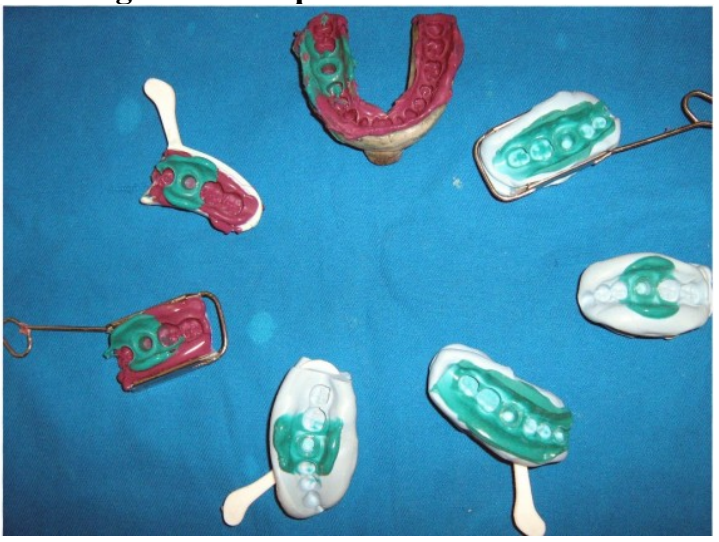

Figure IV- Impressions

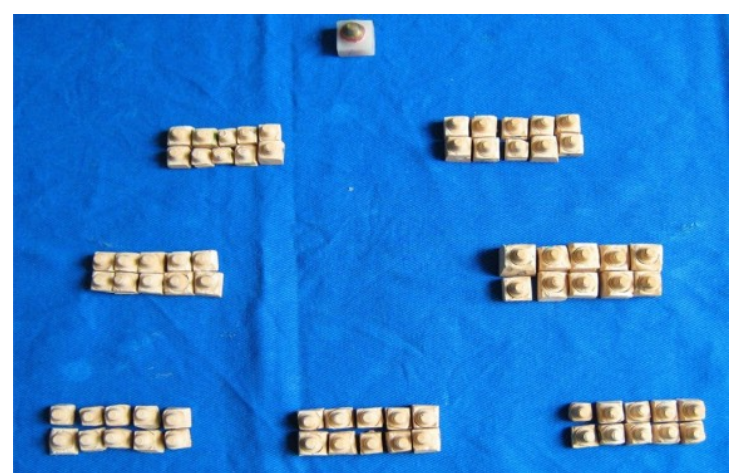

Figure V- The Dies 


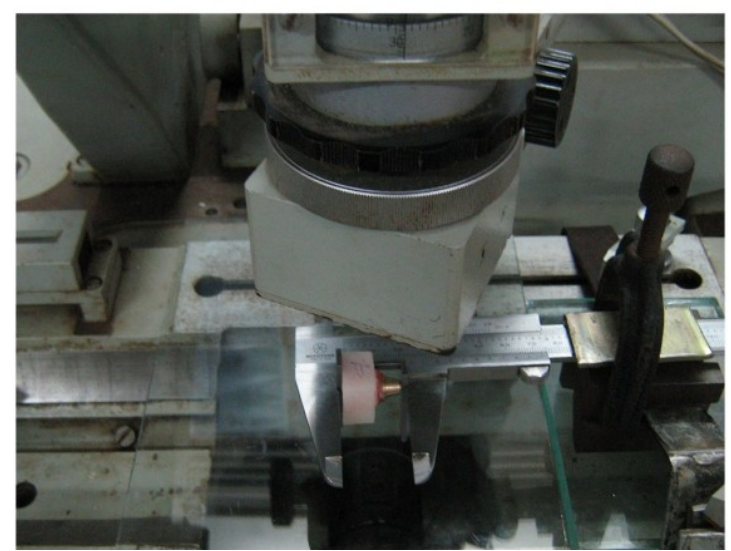

Figure VI- Measuring the dies.

TABLES AND GRAPHS

Table I

\begin{tabular}{|c|c|c|c|c|c|c|c|c|}
\hline & STANDARD & DPPS & DPPD & DPMS & DMPS & DMPD & DMMS & FCMS \\
\hline & 4.9326 & 4.9089 & 4.9012 & 4.9216 & 4.9282 & 4.9283 & 4.9154 & 4.943 \\
\hline & 4.9326 & 4.8963 & 4.9189 & 4.9301 & 4.9282 & 4.9269 & 4.9287 & 4.9401 \\
\hline & 4.9326 & 4.9126 & 4.9129 & 4.9024 & 4.9271 & 4.9276 & 4.9117 & 4.9398 \\
\hline & 4.9326 & 4.8802 & 4.7812 & 4.9117 & 4.9278 & 4.9063 & 4.8991 & 4.9423 \\
\hline & 4.9326 & 4.9085 & 4.9035 & 4.9521 & 4.9185 & 4.9282 & 4.9061 & 4.9444 \\
\hline & 4.9326 & 4.9066 & 4.9091 & 4.9292 & 4.9269 & 4.9301 & 4.9122 & 4.9365 \\
\hline & 4.9326 & 4.9063 & 4.9363 & 4.9132 & 4.9201 & 4.9411 & 4.9311 & 4.9285 \\
\hline & 4.9326 & 4.914 & 4.9242 & 4.9231 & 4.9281 & 4.9098 & 4.9296 & 4.9339 \\
\hline & 4.9326 & 4.9089 & 4.9189 & 4.9123 & 4.9289 & 4.9263 & 4.9243 & 4.9478 \\
\hline & 4.9326 & 4.9062 & 4.9301 & 4.9362 & 4.9062 & 4.9298 & 4.9305 & 4.9521 \\
\hline MEAN & 4.9326 & 4.9048 & 4.9036 & 4.9231 & 4.9240 & 4.9254 & 4.9188 & 4.9408 \\
\hline
\end{tabular}

Occluso-Gingival Measurement (OG) in mm.

Table II

Mesio-Distal Measurement (MD) in mm

\begin{tabular}{|c|c|c|c|c|}
\hline & & & & FCMS \\
\hline & & & & 8.1212 \\
\hline & & & & 8.1204 \\
\hline & & & & 8.1191 \\
\hline & & & & 8.1298 \\
\hline & & & & 8.1331 \\
\hline & & & & 8.1398 \\
\hline & & & & 8.1179 \\
\hline & & & & 8.1185 \\
\hline & & & & 8.1154 \\
\hline & & & & 8.1262 \\
\hline AN & 1234 & 1268 & 279 & 8.1241 \\
\hline
\end{tabular}


Table III

Bucco-Lingual Measurement (BL) in $\mathrm{mm}$.

\begin{tabular}{|c|c|c|c|c|c|c|c|c|}
\hline & STANDARD & DPPS & DPPD & DPMS & DMPS & DMPD & DMMS & FCMS \\
\hline & 8.1005 & 8.1224 & 8.1221 & 8.1142 & 8.1236 & 8.1211 & 8.1065 & 8.1131 \\
\hline & 8.1005 & 8.1215 & 8.1372 & 8.1325 & 8.1269 & 8.1125 & 8.1112 & 8.1023 \\
\hline & 8.1005 & 8.1316 & 8.1244 & 8.1191 & 8.1285 & 8.1134 & 8.1009 & 8.1195 \\
\hline & 8.1005 & 8.1296 & 8.1272 & 8.1121 & 8.1192 & 8.1321 & 8.1123 & 8.1096 \\
\hline & 8.1005 & 8.1328 & 8.1184 & 8.1423 & 8.1246 & 8.1243 & 8.1251 & 8.1122 \\
\hline & 8.1005 & 8.1267 & 8.1193 & 8.1042 & 8.1252 & 8.1106 & 8.1188 & 8.1031 \\
\hline & 8.1005 & 8.1206 & 8.1258 & 8.1092 & 8.1212 & 8.1212 & 8.1052 & 8.1079 \\
\hline & 8.1005 & 8.1304 & 8.1412 & 8.1103 & 8.1168 & 8.1323 & 8.1201 & 8.1201 \\
\hline & 8.1005 & 8.1239 & 8.1241 & 8.1124 & 8.1221 & 8.1268 & 8.1162 & 8.1044 \\
\hline & 8.1005 & 8.1323 & 8.1278 & 8.1068 & 8.1247 & 8.1124 & 8.1009 & 8.1124 \\
\hline MEAN & 8.1005 & 8.1271 & 8.1267 & 8.1163 & 8.1232 & 8.1206 & 8.1117 & 8.1104 \\
\hline
\end{tabular}

Table IV

Standard Deviation for OG, BL And MD, Measurement for all the working Dies

\begin{tabular}{|l|c|c|c|}
\hline & OG & MD & BL \\
\hline DPPS & $\mathbf{. 0 0 0 8 5 8}$ & $\mathbf{. 0 0 0 1 8 9}$ & $\mathbf{0 0 0 7 3 2}$ \\
\hline DPPD & $\mathbf{. 0 0 2 6 1 6}$ & .000135 & .000737 \\
\hline DPMS & .000276 & .000266 & .000381 \\
\hline DMPS & $\mathbf{. 0 0 0 1 2 1}$ & $\mathbf{. 0 0 0 1 5 4}$ & $\mathbf{0 0 0 5 3 0}$ \\
\hline DMPD & .000143 & $\mathbf{. 0 0 0 1 2 7}$ & $\mathbf{. 0 0 0 4 6 7}$ \\
\hline DMMS & $\mathbf{. 0 0 0 3 0 7}$ & $\mathbf{. 0 0 0 2 2 2}$ & $\mathbf{. 0 0 0 1 8 8}$ \\
\hline FCMS & $\mathbf{. 0 0 0 1 0 9}$ & $\mathbf{. 0 0 0 2 3 4}$ & $\mathbf{. 0 0 0 0 1 3 5}$ \\
\hline
\end{tabular}

Table V

Summary of all effects in OG, MD \& BL values Results of ANOVA

\begin{tabular}{|c|c|c|c|c|c|c|c|}
\hline & df effect & MS effect & df error & MS error & F - level \\
\hline OG & $\mathbf{7}$ & $\mathbf{0 . 0 0 1 6 0 7}$ & $\mathbf{7 2}$ & $\mathbf{0 . 0 0 0 3 2 7}$ & $\mathbf{4 . 9 2 1 3 6 9}$ & $\mathbf{0 . 0 0 0 1 3 9}$ \\
\hline MD & $\mathbf{7}$ & $\mathbf{0 . 0 0 0 2 0 6}$ & $\mathbf{7 2}$ & $\mathbf{4 . 4 9 E}-\mathbf{0 5}$ & $\mathbf{4 . 5 8 6 3 7 7}$ \\
\hline BL & $\mathbf{7}$ & $\mathbf{0 . 0 0 0 8 5}$ & $\mathbf{7 2}$ & $\mathbf{5 . 1 2 E - 0 5}$ & $\mathbf{1 6 . 6 0 4 2 3}$ & $\mathbf{7 . 7}$ \\
\hline
\end{tabular}

Table VI

Post Hoc Tests for OG values (p- value $<0.005$ )

\begin{tabular}{|c|c|c|c|c|c|c|c|c|}
\hline & STANDARD & DPPS & DPPD & DPMS & DMPS & DMPD & DMMS & FCMS \\
\hline STANDARD & & $\mathbf{0 . 1 2 6 5 1 9}$ & $\mathbf{0 . 0 9 3 4 4 6}$ & $\mathbf{0 . 9 8 5 9 0 2}$ & $\mathbf{0 . 9 9 1 7 7 5}$ & $\mathbf{0 . 9 9 7 3 6 8}$ & $\mathbf{0 . 8 9 1 7 0 3 5}$ & $\mathbf{0 . 9 9 3 6 6 5}$ \\
\hline DPPS & $\mathbf{0 . 1 2 6 5 1 9}$ & & $\mathbf{1}$ & $\mathbf{0 . 6 4 2 4 4 3}$ & $\mathbf{0 . 5 8 8 2 4 7}$ & $\mathbf{0 . 4 9 0 9 2 8}$ & $\mathbf{0 . 8 8 0 4 2 7 8}$ & 0.011503 \\
\hline DPPD & $\mathbf{0 . 0 9 3 4 4 6}$ & $\mathbf{1}$ & & $\mathbf{0 . 5 6 0 4 9 6}$ & $\mathbf{0 . 5 0 5 7 0 1}$ & $\mathbf{0 . 4 1 1 0 2}$ & $\mathbf{0 . 8 2 5 6 4 9 9}$ & 0.00757 \\
\hline DPMS & $\mathbf{0 . 9 8 5 9 0 2}$ & $\mathbf{0 . 6 4 2 4 4 3}$ & $\mathbf{0 . 5 6 0 4 9 6}$ & & $\mathbf{0}$ & $\mathbf{0 . 9 9 9 9 9 9}$ & $\mathbf{0 . 9 9 9 9 0 5 5}$ & $\mathbf{0 . 6 8 7 2 9 7}$ \\
\hline DMPS & $\mathbf{0 . 9 9 1 7 7 5}$ & $\mathbf{0 . 5 8 8 2 4 7}$ & $\mathbf{0 . 5 0 5 7 0 1}$ & $\mathbf{1}$ & & $\mathbf{0 . 9 9 9 7 0 0 7}$ & $\mathbf{0 . 7 3 7 5 1 5}$ \\
\hline DMPD & $\mathbf{0 . 9 9 7 3 6 8}$ & $\mathbf{0 . 4 9 0 9 2 8}$ & $\mathbf{0 . 4 1 1 0 2}$ & $\mathbf{0 . 9 9 9 9 9 9}$ & $\mathbf{1}$ & $\mathbf{0 . 9 9 8 4 8 1 8}$ & $\mathbf{0 . 8 1 7 6 1 8}$ \\
\hline DMMS & $\mathbf{0 . 8 9 1 7 0 4}$ & $\mathbf{0 . 8 8 0 4 2 8}$ & $\mathbf{0 . 8 2 5 6 5}$ & $\mathbf{0 . 9 9 9 9 0 6}$ & $\mathbf{0 . 9 9 9 7 0 1}$ & $\mathbf{0 . 9 9 8 4 8 2}$ & \\
\hline FCMS & $\mathbf{0 . 9 9 3 6 6 5}$ & 0.011503 & 0.00757 & $\mathbf{0 . 6 8 7 2 9 7}$ & $\mathbf{0 . 7 3 7 5 1 5}$ & $\mathbf{0 . 8 1 7 6 1 8}$ & $\mathbf{0 . 4 0 0 8 8 6 5}$ \\
\hline
\end{tabular}


Table VII

Post Hoc Tests for MD values ( $\mathrm{p}$ - value $<0.005$ )

\begin{tabular}{|c|c|c|c|c|c|c|c|c|}
\hline & STANDARD & DPPS & DPPD & DPMS & DMPS & DMPD & DMMS & FCMS \\
\hline STANDARD & & 0.065754 & 0.140692 & 0.005703 & 0.097263 & 0.192788 & 0.007035 & 0.011297 \\
\hline DPPS & 0.065754 & & 0.999992 & 0.995784 & 1 & 0.999893 & 0.997454 & 0.999366 \\
\hline DPPD & 0.140692 & 0.999992 & & 0.970325 & 1 & 1 & 0.978514 & 0.990923 \\
\hline DPMS & 0.005703 & 0.995784 & 0.970325 & & 0.987667 & 0.9422 & 1 & 1 \\
\hline DMPS & 0.097263 & 1 & 1 & 0.987667 & & 0.999994 & 0.991738 & 0.997199 \\
\hline DMPD & 0.192788 & 0.999893 & 1 & 0.9422 & 0.999994 & & 0.955732 & 0.978183 \\
\hline DMMS & 0.007035 & 0.997454 & 0.978514 & 1 & 0.991738 & 0.955732 & & 1 \\
\hline FCMS & 0.011297 & 0.999366 & 0.990923 & 1 & 0.997199 & 0.978183 & 1 & \\
\hline
\end{tabular}

Table VIII

Post Hoc Tests for BL values ( $\mathrm{p}$ - value $<0.005$ )

\begin{tabular}{|c|c|c|c|c|c|c|c|c|}
\hline & STANDARD & DPPS & DPPD & DPMS & DMPS & DMPD & DMMS & FCMS \\
\hline STANDARD & & $1.29 \mathrm{E}-08$ & $2.2 \mathrm{E}-08$ & 0.002826 & $1.48 \mathrm{E}-06$ & $3.02 \mathrm{E}-05$ & $\mathbf{0 . 1 0 9 4 7 3}$ & $\mathbf{0 . 2 2 5 1 1 4}$ \\
\hline DPPS & $1.29 \mathrm{E}-08$ & & $\mathbf{1}$ & $\mathbf{0 . 1 3 5 6 2 3}$ & $\mathbf{0 . 9 8 1 5 6 6}$ & $\mathbf{0 . 7 6 0 7 9 8}$ & 0.003918 & 0.001173 \\
\hline DPPD & $2.2 \mathrm{E}-08$ & $\mathbf{1}$ & & $\mathbf{0 . 1 7 3 9 0 5}$ & $\mathbf{0 . 9 9 0 7 6 9}$ & $\mathbf{0 . 8 1 9 7 1 8}$ & 0.005798 & 0.001786 \\
\hline DPMS & 0.002826 & $\mathbf{0 . 1 3 5 6 2 3}$ & $\mathbf{0 . 1 7 3 9 0 5}$ & & $\mathbf{0 . 6 9 0 0 5 4}$ & $\mathbf{0 . 9 6 5 3 5 1}$ & $\mathbf{0 . 9 5 4 1 6 5}$ \\
\hline DMPS & $1.48 \mathrm{E}-06$ & $\mathbf{0 . 9 8 1 5 6 6}$ & $\mathbf{0 . 9 9 0 7 6 9}$ & $\mathbf{0 . 6 9 0 0 5 4}$ & & $\mathbf{0 . 9 9 8 4 4 7}$ & $\mathbf{0 . 0 8 8 0 3 4}$ & $\mathbf{0 . 0 3 6 2 1}$ \\
\hline DMPD & $3.02 \mathrm{E}-05$ & $\mathbf{0 . 7 6 0 7 9 8}$ & $\mathbf{0 . 8 1 9 7 1 8}$ & $\mathbf{0 . 9 6 5 3 5 1}$ & $\mathbf{0 . 9 9 8 4 4 7}$ & & $\mathbf{0 . 3 6 1 4 9 9}$ \\
\hline DMMS & $\mathbf{0 . 1 0 9 4 7 3}$ & 0.003918 & 0.005798 & $\mathbf{0 . 9 5 4 1 6 5}$ & $\mathbf{0 . 0 8 8 0 3 4}$ & $\mathbf{0 . 3 6 1 4 9 9}$ & $\mathbf{0 . 1 9 7 3 1}$ \\
\hline FCMS & $\mathbf{0 . 2 2 5 1 1 4}$ & 0.001173 & 0.001786 & $\mathbf{0 . 8 4 7 7 8 5}$ & $\mathbf{0 . 0 3 6 2 1}$ & $\mathbf{0 . 1 9 7 3 1}$ & $\mathbf{0 . 9 9 9 9 8 8}$ \\
\hline
\end{tabular}

Graph I- Categorised plot for OG values

Graph II- Categorised plot for MD values
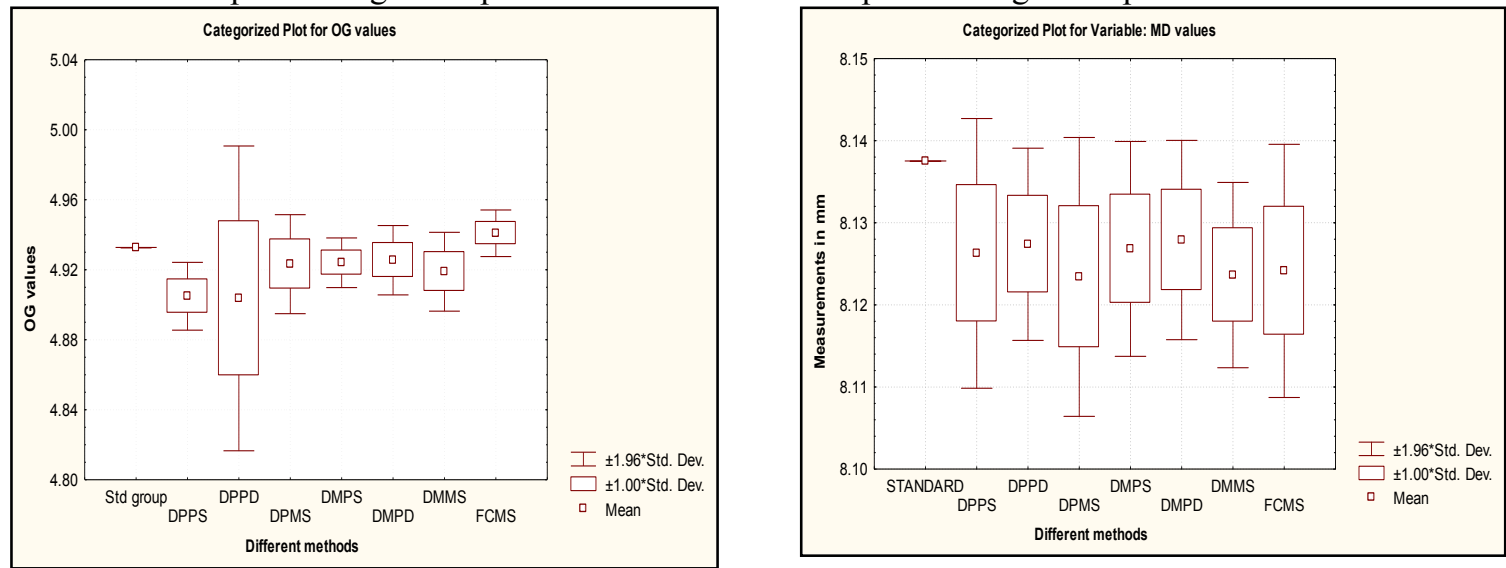

Graph III- Categorised plot for BL values

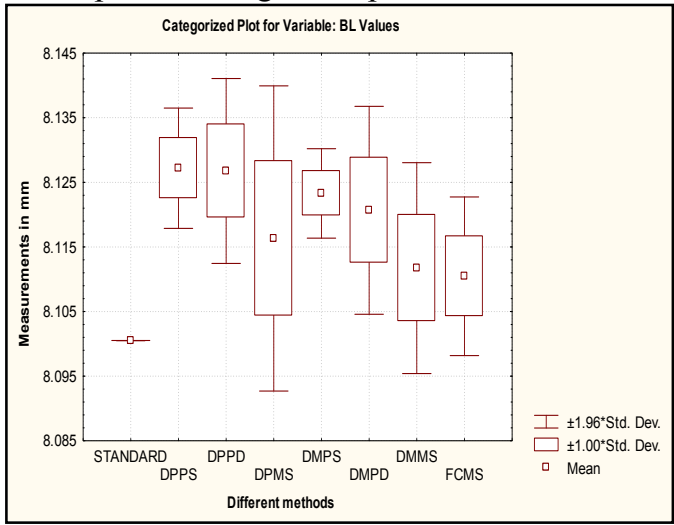

